\title{
Photo Quiz
}

(For answer and discussion, see page 3447 in this issue [doi:10.1128/JCM.r00875-11])

\section{A 30-Year-Old Male with a 4-Week History of Shortness of Breath, Productive Cough, and Weight Loss}

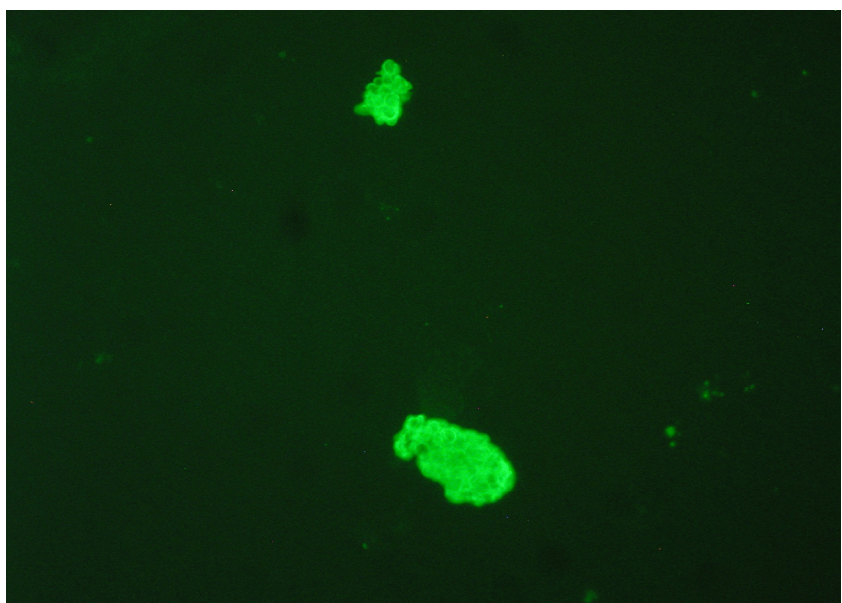

FIG. 1. Pneumocystis jirovecii (formerly carinii) DFA (MeriFluor Pneumocystis; Meridian Bioscience, Cincinnati, OH) bronchoalveolar lavage specimen. Total magnification, $\times 600$.

During the period of heightened severe acute respiratory syndrome (SARS) coronavirus vigilance in early 2005, a 30year-old male with an 8-year history of human immunodeficiency virus (HIV) infection presented to the emergency department with complaints of shortness of breath, productive cough, night sweats, and poor oral intake. He also reported a 10- to 20-pound weight loss. His travel history was noncontributory. Examination of his social history revealed tobacco, alcohol, and illicit drug consumption. The patient had shown a negative purified-protein-derivative (PPD) skin test result during a recent incarceration. The man was employed as a security guard and indicated that individuals from China and India passed his post on a regular basis.

Physical examination produced vital signs indicating pyrexia (maximum temperature $\left[T_{\max }\right], 101^{\circ} \mathrm{F}$ ), tachypnea (respiratory rate, 22), and slight tachycardia (heart rate, 95). His blood pressure was $103 / 63$. The patient demonstrated bilateral breath sounds and showed normal cardiac examination results. Laboratory studies indicated hypoxia (partial $\mathrm{O}_{2}$ pressure $\left[\mathrm{pO}_{2}\right], 87 \%$ on room air), compromised nutritional status (serum albumin concentration, $2.9 \mathrm{~g} / \mathrm{dl}$ ), and advanced HIV disease (leukocyte count, 3,700 cells/ $\mu$;
$\mathrm{CD}^{+}$T-lymphocyte count, 17 cells $/ \mathrm{mm}^{3}$ ). A chest X-ray revealed bilateral diffuse infiltrates.

Direct examination of expectorated sputum via Gram, acidfast fluorochrome, calcofluor white, and respiratory virus direct fluorescent-antibody assay (DFA) staining techniques yielded no significant findings. DFA staining of this specimen for Pneumocystis jirovecii (formerly carinii) was negative. The patient underwent a bronchoalveolar lavage (BAL) the following day, and a diagnosis was made on the basis of the results of the DFA (slide illustrated in Fig. 1).

Erik Munson*

Wheaton Franciscan Laboratory 11020 West Plank Court

Suite 100

Wauwatosa, Wisconsin 53226

Mary Paul

Wheaton Franciscan Laboratory

Wauwatosa, Wisconsin 53226

*Phone: (414) 256-1479

Fax: (414) 256-5566

E-mail: Erik.Munson@wfhc.org 